\title{
Saúde e trabalho de
}

\section{residentes multiprofissionais}

\author{
Health and Work of the \\ Multiprofessional Residents
}

\section{Salud y trabajo de residentes \\ multiprofesionales}

\author{
Jéssica Santos Rocha, Esp.'; \\ Raquel Aparecida Casarotto, $\mathrm{PhD}^{1}$; \\ Ana Carolina Basso Schmitt, $\mathrm{PhD}^{2 *}$
}

Recibido: 10 de octubre de 2017 / Aceptado: 21 de mayo de 2018

Dor: http://dx.doi.org/10.12804/revistas.urosario.edu.co/revsalud/a.7265

Para citar este artigo: Rocha SJ, Casarotto AR, Schmitt BAC. Saúde e trabalho de residentes multiprofissionais. Rev Cienc Salud.

2018;16(3):447-62. doi http://dx.doi.org/10.12804/revistas.urosario.edu.co/revsalud/a.7265

\section{Resumo}

Introdução: O Programa de Residência Multiprofissional em Saúde (RM) é, no Brasil, uma das estratégias para reorganização do serviço de saúde. Tal programa foi baseado nas Residências Médicas que são alvo de apontamentos críticos relacionados à supervisão e à situação de saúde dos residentes. Na literatura são encontrados poucos estudos que investiguem a saúde dos residentes multiprofissionais. O objetivo do estudo foi analisar e correlacionar a qualidade de vida (Qv), estresse e satisfação com 0 trabalho de tais residentes. Materiais e métodos: Estudo transversal, com 42 residentes de 2014-2015 de cinco programas de residência multiprofissional dos três níveis assistenciais. Com questionário online, foi estimada a prevalência de estresse e construídas regressões lineares múltiplas para verificar a contribuição das variáveis sociodemográficas, ocupacionais, estresse e da satisfação com o trabalho nos domínios da Qv (significância de $5 \%$ ). Resultado: Verificou-se que $78.9 \%$ dos residentes apresentava estresse. Os residentes estavam parcialmente satisfeitos em relação à satisfação com o trabalho de modo geral. Quanto à Qv, os valores apresentados em todos os domínios foram inferiores aos encontrados na população brasileira em geral. Conclusão: A satisfação parcial com o trabalho envolve o relacionamento com os pares, esgotamento profissional e grau de flexibilidade e de liberdade na participação do residente no processo de trabalho. A prevalência do estresse nos residentes multiprofissionais foi alta, ele e a mobilidade urbana contribuíram negativamente para a Qv, por outro lado, ter atividade de lazer teve influência positiva na Qv.

Palavras-chave: saúde do trabalhador, qualidade de vida, estresse profissional.

1 Departamento de Fisioterapia, Fonoaudiologia e Terapia Ocupacional, Faculdade de Medicina, Universidade de São Paulo.

2 Departamento de Fisioterapia, Fonoaudiologia e Terapia Ocupacional da FMUsP, São Paulo, Brasil.

Autora para correspondência: carolinaschmitt@usp.br 


\section{Resumen}

Introducción: el Programa de Residencia Multiprofesional en Salud (RM) es una de las estrategias en Brasil para la reorganización del servicio de salud. Tal programa fue basado en las Residencias Médicas que son blanco de críticas relacionadas con la supervisión y a la situación de salud de los residentes. Son encontrados en la literatura pocos estudios que investiguen la salud de los residentes multiprofesionales. El objetivo de este estudio fue analizar y correlacionar la calidad de vida (Qv), estrés y satisfacción con el trabajo de tales residentes. Materiales y métodos: estudio transversal, con 42 residentes de 2014-2015 de cinco programas de residencia multiprofesional de los tres niveles asistenciales. Con cuestionario online, fue estimada la prevalencia de estrés y construidas regresiones lineales múltiples para verificar la contribución de las variables sociodemográficas, ocupacionales, estrés y de satisfacción con el trabajo en los dominios de la qv (significancia de $5 \%$ ). Resultado: se verificó que $78.9 \%$ de los residentes presentaba estrés. Los residentes estaban parcialmente satisfechos en relación con la satisfacción con el trabajo de manera general. En cuanto a la Qv, los valores presentados en todos los dominios fueron inferiores a los encontrados en la población brasilera en general. Conclusión: la satisfacción parcial con el trabajo involucra el relacionamiento los pares, agotamiento profesional y grado de flexibilidad y de libertad en la participación del residente en el proceso de trabajo. La prevalencia del estrés en los residentes multiprofesionales fue alta y junto con la movilidad urbana, contribuyen negativamente para la Qv; por otro lado, tener actividad de ocio tuvo influencia positiva.

Palabras clave: salud del trabajador, calidad de vida, estrés profesional.

\section{Abstract}

Introduction: The Multiprofessional Residency Program (RM) is one of the strategies in Brazil to reorganize the health service. This program was based on the Medical Residencies that are the target of critical notes related to supervision and the residents' health situation. There are few studies in the literature that investigate the health of multiprofessional residents. The aim of this study is to analyze and relate the quality of life (QOL), stress and satisfaction with the work of multiprofessional residents. Materials and methods: This crosssectional study is with 42 residents of 2014-2015 from five programs of multidisciplinary residency of three levels of care. With online survey, it estimated the prevalence of stress and built multiple linear regressions to verify the contribution of sociodemographic, occupational, stress and job satisfaction variables in the domains of QoL (5\% significance). Results: It was found that $78.9 \%$ of the resident had stress. Residents were partially satisfied in relation to satisfaction with the general work. About the QoL, the amounts presented in all areas were lower than those found in the Brazilian population in general. Conclusion: The partial satisfaction with the work involves the relationship with the peers, professional exhaustion and degree of flexibility and freedom in the resident's participation in the work process. The prevalence of stress in multiprofessional residents was high, the Stress and urban mobility contributed to be negative to the QoL; on the other hand the leisure had a positive influence.

Keywords: Occupational health, quality of life, burnout professional.

\section{Introdução}

$A_{\text {recursos humanos capacitados para atender às necessidades do sistema de saúde brasi- }}^{\text {formaco do profissional de saúde é objeto de reflexões, visto que há necessidade de }}$ leiro com todas suas particularidades. 
Para responder a tal desafio, a formação dos profissionais de saúde atuantes no sistema de saúde vigente no Brasil —Sistema Único de Saúde (sus)—, é objeto de frequentes estudos e reformulações. Desde o final da década de 1980, propostas de mudança na formação em saúde vêm sendo discutidas para qualificação dos recursos humanos para o bem cuidar da população. Como estratégia de reorganização dos serviços públicos com base nos preceitos do sus, em novembro de 2005, foi instituído o Programa de Residência Multiprofissional em Saúde, com foco na produção de condições necessárias para a mudança do modelo assistencial restritivo de atenção à saúde (1). As residências multiprofissionais (RM) e em área profissional da saúde abrangem as profissões Biomedicina, Ciências Biológicas, Educação Física, Enfermagem, Farmácia, Fisioterapia, Fonoaudiologia, Medicina Veterinária, Nutrição, Odontologia, Psicologia, Serviço Social e Terapia Ocupacional (2).

O modelo biomédico, ainda pauta a formação dos demais profissionais da saúde de forma fragmentada e dificulta a formação generalista sob o ponto de vista de entendimento da intervenção e dos condicionantes do processo saúde-doença da população, criando obstáculos para a integralidade da assistência (3). Torna-se necessário, então, que o processo de formação esteja articulado com o mundo do trabalho e que o profissional seja preparado para trabalhar em equipe multiprofissional, para proporcionar a integralidade da atenção e a atuação, de acordo com as diretrizes e os princípios do sus.

As RM, segundo Feuerwerker, foram inspiradas na residência médica, e têm como objetivo a articulação dos conhecimentos, muitas vezes fragmentados e desarticulados, adquiridos na graduação, com a complexidade dos determinantes que se inter-relacionam na vida e no cuidado à saúde da população (3).

A residência médica foi implantada no Brasil em 1944-1945, e é alvo de apontamentos críticos relacionados, sobretudo, à adequada oferta de supervisão qualificada e aos potenciais efeitos deletérios da carga horária de trabalho e da privação do sono (4). Experiências com residências médicas têm evidenciado que o treinamento pode ter efeitos deletérios à saúde, bem-estar e na qualidade de vida pessoal do profissional, bem como ser prejudicial à sua sensibilidade em relação aos pacientes, no seu desempenho profissional e acadêmico $(5,6)$. Dada a recente implementação dos programas de RM, são encontrados poucos estudos na literatura, que investiguem a saúde dos residentes multiprofissionais.

Diante da contextualização recente dos programas de RM e de sua perspectiva como uma política indutora para qualificação do sistema de saúde brasileiro, é importante ter atenção à saúde do residente, uma vez que ele como protagonista neste processo é exposto à demanda de trabalho e expectativa de aprendizagem qualificada. Assim, o objetivo do estudo foi analisar e correlacionar a qualidade de vida, estresse e satisfação com o trabalho de residentes multiprofissionais de uma universidade pública do estado de São Paulo. 


\section{Materiais e métodos}

Trata-se de um estudo transversal com residentes multiprofissionais de uma universidade pública do estado de São Paulo, matriculados nos anos de 2014 e 2015, do primeiro e segundo ano de cinco programas de RM com 90 vagas disponíveis, com inserção na atenção primária à saúde, na média e na alta complexidade. Foram excluídos os residentes em licença de qualquer natureza no período da coleta de dados. Esta foi realizada, inicialmente, via contato por e-mail com os representantes dos residentes de cada programa, os quais disponibilizaram os endereços eletrônicos dos indivíduos que aceitaram passar seus contatos, totalizando 61. Apenas 2 residentes negaram-se a disponibilizar seus contatos. Assim, os residentes foram convidados, por três tentativas em uma periodicidade de 15 dias, a participar da pesquisa, por meio de um questionário online (figura 1).

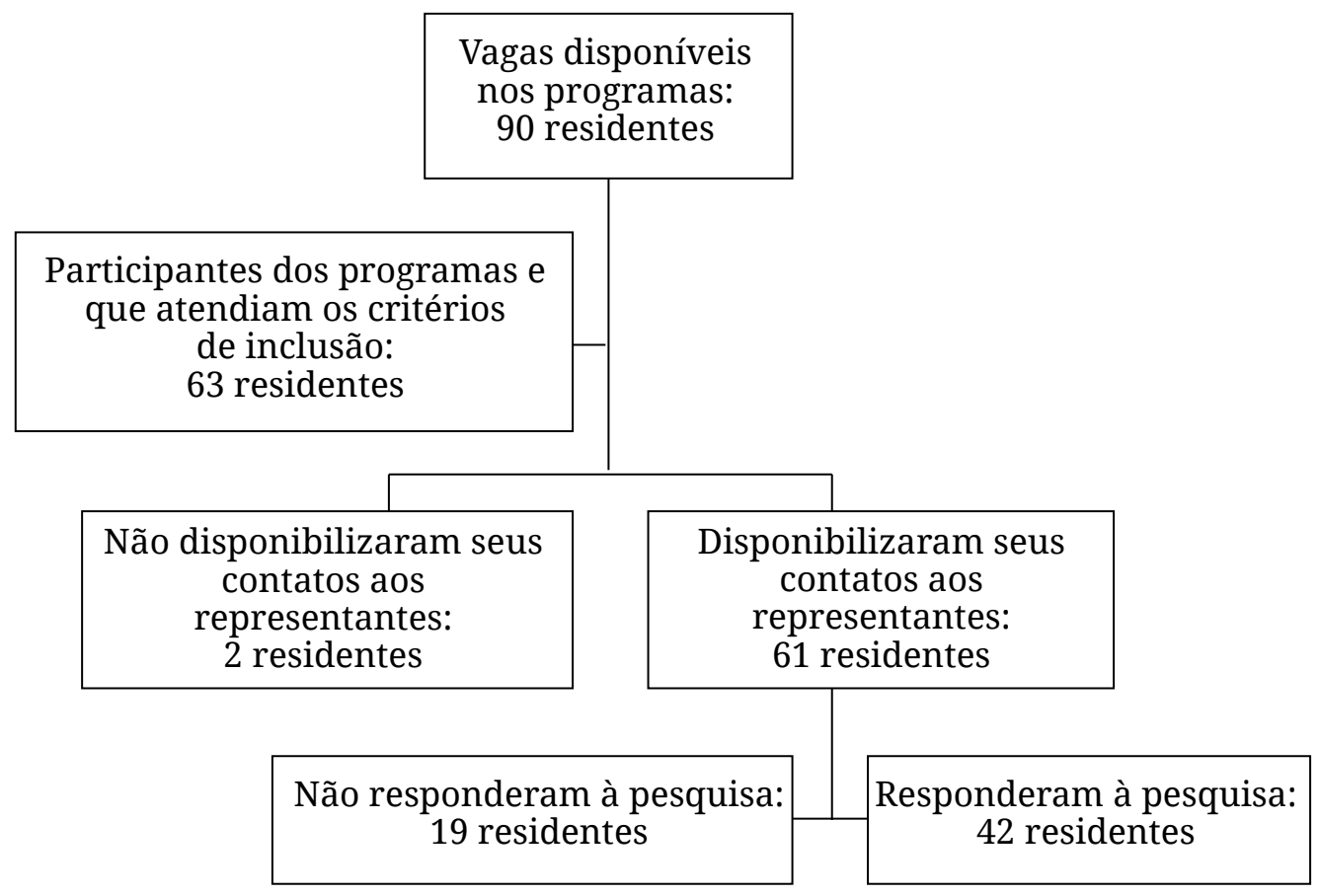

Figura 1. Fluxograma de seleção da amostra estudada. São Paulo, 2015

Foram avaliados: perfil sociodemográfico e ocupacional, qualidade de vida, estresse e satisfação com o trabalho.

O perfil sociodemográfico e ocupacional foi composto por questionamento sobre sexo, idade, estado civil, cor/raça, ocupação (de acordo com a Classificação Brasileira de Ocupações), prática de atividade física (questionário internacional de atividade física versão curta - IPAQ CURTo), tempo e disposição para o lazer, atividades profissionais fora da residência, formação prévia (outras pós-graduações), meios de transporte para chegar ao local de trabalho, atividades remuneradas além da RM (7-9). 
Para a análise da qualidade de vida foi utilizado o whoQoL-bref, que valoriza a percepção individual da pessoa. Trata-se de instrumento curto, de rápida aplicação e recomendado tanto para pessoas com algum tipo de doença como para as saudáveis (10). Foi validado para português brasileiro, ou seja, pt/br, e apresentou boa consistência interna, validade discriminante, validade concorrente, validade de conteúdo e confiabilidade teste-reteste (11). É composto por 26 questões para avaliar qualidade de vida em diversos grupos e situações. A primeira refere-se à qualidade de vida de modo geral e a segunda, à satisfação com a própria saúde, o que gera o escore da qualidade de vida geral (10). As outras 24 perguntas formam os domínios físico, psicológico, relações sociais e meio ambiente (11). A cada item do questionário as respostas variam de 1 a 5 , de forma que quanto mais próxima de 5 , melhor a qualidade de vida. A soma dos escores finais médios de cada domínio são calculados por uma sintaxe, resultando em escore que pode variar entre 4 e 20 (12). Utilizando-se a correspondência entre o wHOQOL-100 e o wHOQOL-bref, o resultado mais próximo de 100 representa melhor qualidade de vida (13-15).

Para a avaliação do estresse no trabalho foi utilizado Inventário de Sintomas de Stress para Adultos (ISsL), que permite diagnosticar o estresse, a prevalência dos sintomas (físicos ou psicológicos) e as fases de alerta, resistência e exaustão com alta confiabilidade (coeficiente alfa de 0.9121)(16,17). O IssL possui 37 itens de natureza somática e 19 de natureza psicológica, compondo, no total, 53 sintomas divididos em três partes, que se referem às fases do estresse: alerta - o primeiro sintoma experimentado nas últimas 24 horas ( $\geq 7$ pontos); resistência - sintomas da última semana ( $\geq 4$ pontos); exaustão - sintomas do último mês ( $\geq 9$ pontos) (18). A correção e a interpretação do IssL foram realizadas por psicólogo (CRP 06/79121) (16).

A satisfação como trabalho foi avaliada por meio da Escala Satisfação no Trabalho do osı - Occupational Stress Indicator de Cooper, Sloan e Williams, com tradução e validação para o português brasileiro, ou seja, $\mathrm{PT} / \mathrm{BR}$, considerada com boa validade para medidas da saúde física e do componente psicossomático (19-21). São utilizados 22 aspectos psicossociais do trabalho, que compõem uma escala de Likert de seis pontos, e não possui um valor de corte definido ou divisão em níveis. A categorização foi feita segundo Martinez (2002) (17). 1. insatisfação: enorme insatisfação e muita insatisfação; 2. satisfação intermediária: alguma insatisfação e alguma satisfação; 3. satisfação: muita satisfação e enorme satisfação. Para melhor compreensão, as questões do osı foram classificadas em seis domínios: comunicação - questões 1 e 10; relacionamento - questões 2, 13, 15 e 18; motivação - questões 5 e 8; realizações - questões $3,4,16,17,19,20$ e 22; perspectivas - questões 6, 7, 12 e 14; cobranças - questões 9, 11 e 21 (22).

Os dados coletados foram analisados por meio do programa sTATA versão 12. Para a análise das variáveis sociodemográficas, ocupacionais, da satisfação com o trabalho e qualidade de vida, foram utilizadas medidas de tendência central e dispersão e porcentagem. Foi 
estimada a prevalência de estresse com intervalo de confiança de $95 \%$. Foram analisadas as correlações e construídas as análises de regressão linear simples para verificar a interferência dos domínios da qualidade de vida com a qualidade de vida geral. Foi feita a correlação de Pearson entre os domínios (físico, psicológico, social e meio ambiente) e o domínio global da qualidade de vida. Por fim, para verificar a contribuição das variáveis sociodemográficas, ocupacionais, estresse e de satisfação com o trabalho nos domínios da qualidade de vida foram construídas regressões lineares múltiplas. O nível de significância adotado foi de $5 \%$.

Somente os residentes multiprofissionais que concordaram com o Termo de Consentimento Livre e Esclarecido participaram do estudo. Atendendo-se às Diretrizes e Normas Regulamentadoras de Pesquisas Envolvendo Seres Humanos do Conselho Nacional de Saúde, o projeto desta pesquisa foi apreciado e aprovado pelo Comitê de Ética em Pesquisa (Protocolo: 041292/2015) (23).

\section{Resultados}

$\mathrm{D}$ os 63 residentes dos programas estudados, foram obtidos 61 contatos de e-mail e 42 residentes multiprofissionais responderam à pesquisa.

Tabela 1. Estatística descritiva das variáveis sociodemográficas da amostra estudada. São Paulo, 2015

\begin{tabular}{|c|c|c|c|c|}
\hline Perfil & Categorias & Descrição & $\mathbf{N}$ & $\%$ \\
\hline \multirow{4}{*}{ Idade (anos) } & & Mínima: 22.9 & 40 & \\
\hline & & Máxima: 35.3 & & \\
\hline & & Média: 25.96 & & \\
\hline & & Desv. Padrão: 2.3 & & \\
\hline \multirow{2}{*}{ Estado civil } & Solteiro & & 40 & 95.2 \\
\hline & Casado & & 2 & 4.8 \\
\hline \multirow{7}{*}{ Sexo } & Feminino & & 34 & 80.9 \\
\hline & Masculino & & 8 & 19.1 \\
\hline & Branca & & 24 & 57.1 \\
\hline & Parda & & 7 & 16.7 \\
\hline & Amarela & & 6 & 14.3 \\
\hline & Preta & & 4 & 9.5 \\
\hline & Não respondeu & & 1 & 2.4 \\
\hline
\end{tabular}




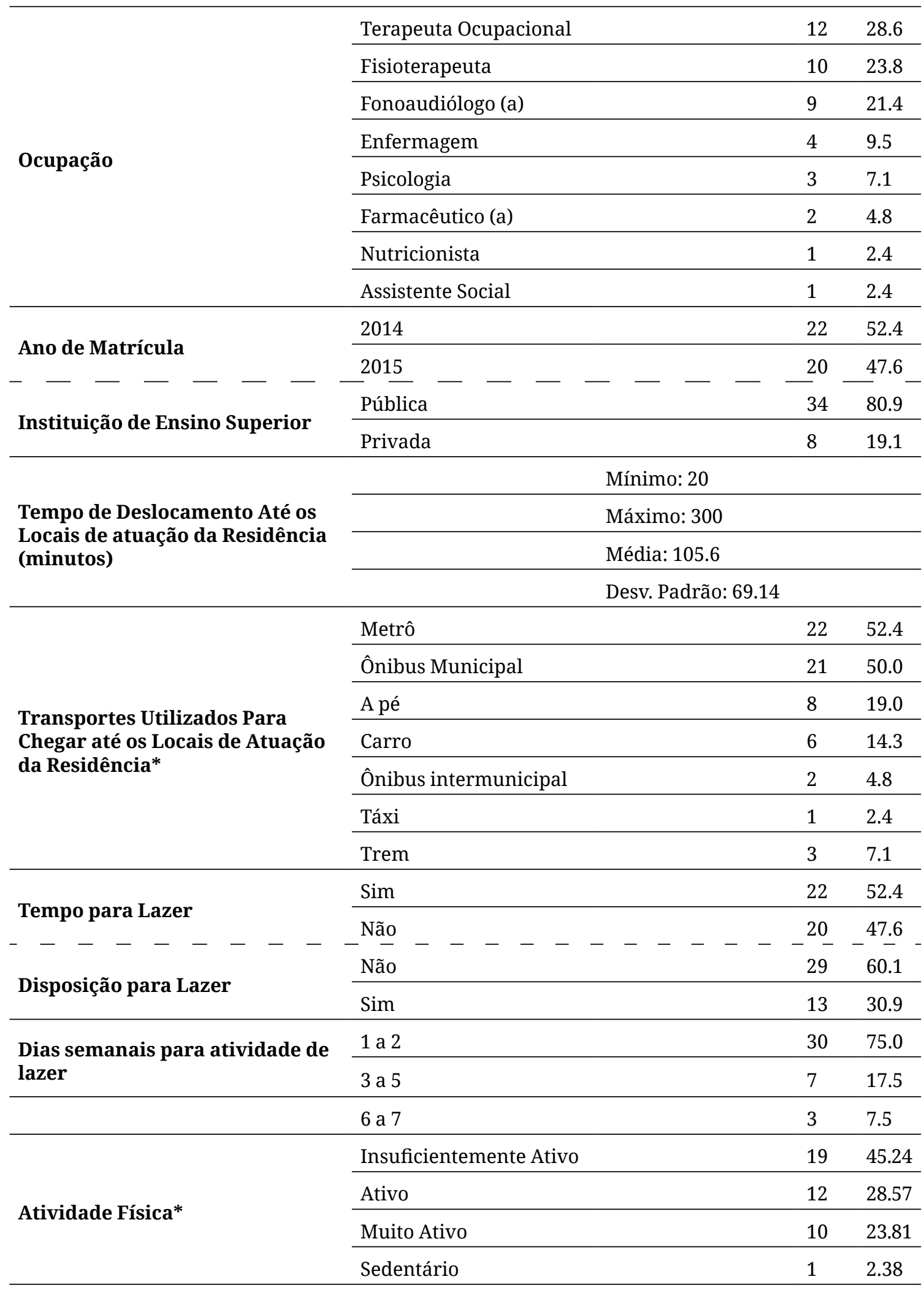


A prevalência de estresse na amostra foi de 78.9\% ( $\mathrm{IC}_{95 \%}: 65.3 \%$ - 92.5\%). A maioria dos residentes com estresse estava na fase de resistência (64.3\%), $9.5 \%$ alerta, $2.4 \%$ exaustão, $9.5 \%$ alerta/resistência e em $14.3 \%$ não foi possível determinar a fase.

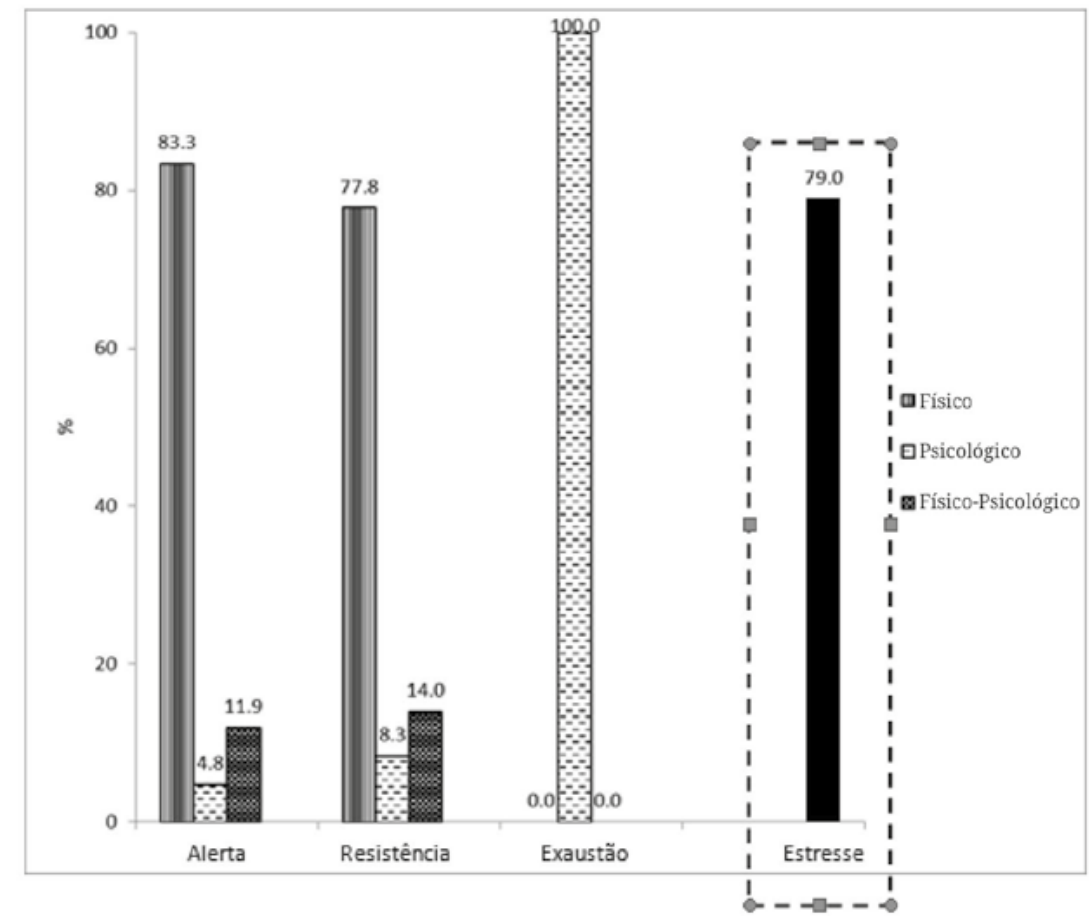

Figura 2. Presença de estresse e sintomas físico, psicológico e físico-psicológico, segundo as fases do estresse dos residentes multiprofissionais, São Paulo - SP, 2015

Os sintomas de estresse prevalentes na fase de alerta foram tensão muscular (81\%), mudança de apetite (54.8\%), insônia (38.1\%), ranger de dentes (35.7\%). Na fase de exaustão, insônia (38.9\%), mudança extrema de apetite (27.8\%), problema dermatológico prolongado (22.2\%), tiques e diarreias (16.7\%). Os sintomas psicológicos na fase de alerta foram entusiasmo súbito (23.8\%) e motivação e iniciar novos projetos (21.4\%). Na fase de resistência foram pensar num único assunto (52.8\%); dúvida de si próprio (50\%), irritabilidade e sensibilidade excessiva (47.2\%). Enquanto na fase de exaustão foram cansaço excessivo (69.4\%), vontade de fugir (58.3\%), e ansiedade (44.4\%).

Para a satisfação com o trabalho (figura 3), os aspectos com os quais há maior proporção de residentes satisfeitos são: o grau em que a organização absorve as potencialidades que julga ter - potencialidades (35.7\%) e a estrutura organizacional da sua residência - estrutura (35\%). Já os aspectos com maior proporção de residentes insatisfeitos foram: relacionamento com outras pessoas nos locais de trabalho em que atua na residência - relacionamento (40.9\%) e o grau de flexibilidade e de liberdade que julga ter em seu trabalho - flexibilidade (40.5\%). 


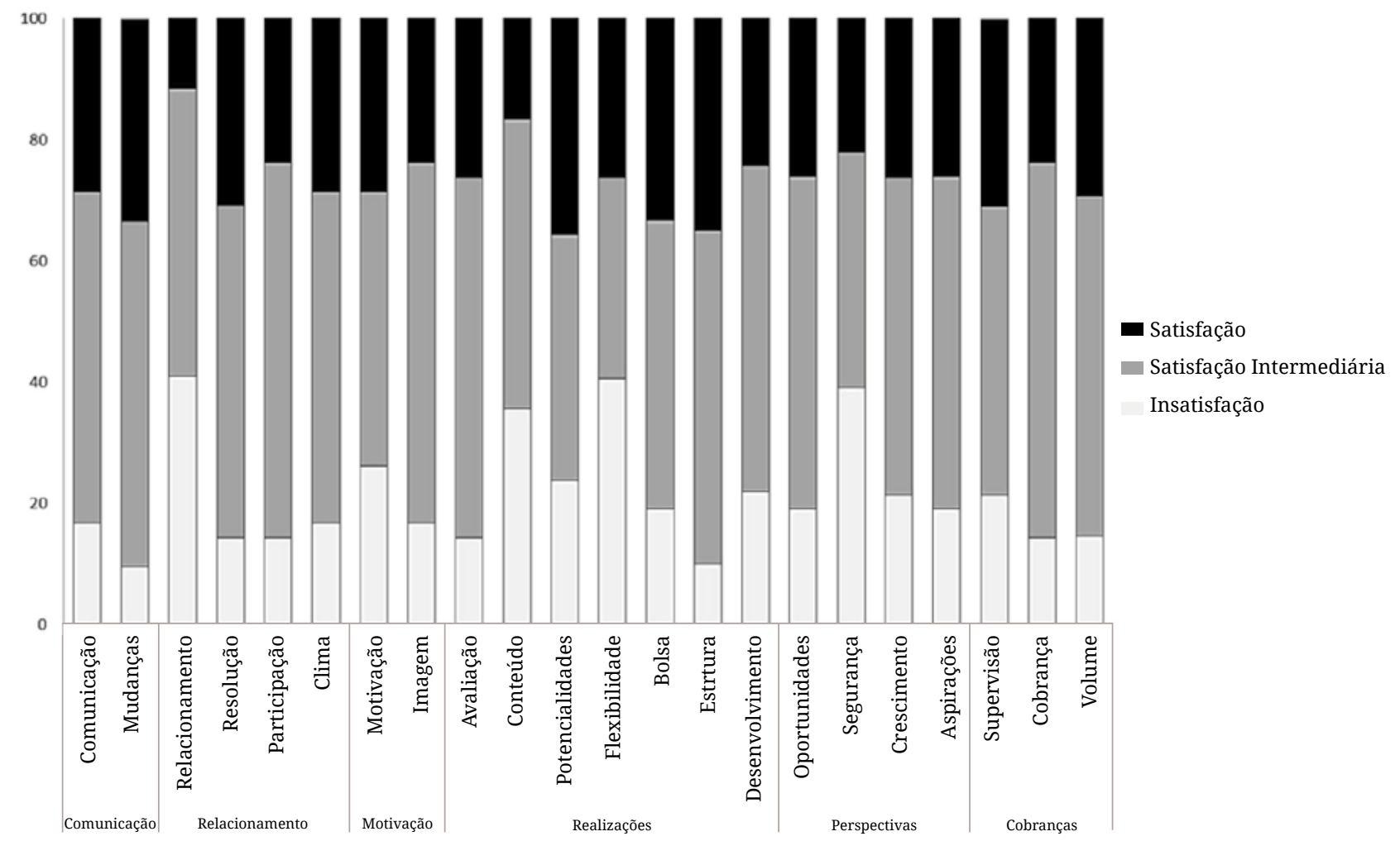

Figura 3. Proporção de residentes multiprofissionais, segundo o grau de satisfação com o trabalho. São Paulo, 2015

Com relação à qualidade de vida, a tabela 2 mostra o domínio psicológico, como sendo 0 melhor aspecto da Qv. Em segundo lugar, tem-se o domínio físico, seguido da qualidade de vida geral, do meio ambiente e do domínio de relações sociais, respectivamente. Na mesma tabela, evidencia-se a influência que todos os domínios da qualidade de vida tiveram contribuição significativa sob o domínio geral ( $\mathrm{p}<.001)$.

Tabela 2. Medidas de tendência central e dispersão, estimativa de correlação de Pearson e análise de regressão linear entre os domínios e a qualidade de vida geral. São Paulo, 2015

\begin{tabular}{|c|c|c|c|c|c|}
\hline \multirow{3}{*}{ Domínio } & \multirow{3}{*}{ Média (dp) } & \multirow{3}{*}{ Mínimo - Máximo } & \multicolumn{3}{|c|}{ Qualidade de vida geral (dependente) } \\
\hline & & & \multirow{2}{*}{$\begin{array}{c}\text { Correlação } \\
\text { (r) }\end{array}$} & \multicolumn{2}{|c|}{ Regressão } \\
\hline & & & & $\mathbf{R}^{2}(\%)$ & $\boldsymbol{\beta}$ \\
\hline Psicológico & $58.2(8.3)$ & $37.5-87.5$ & .71 & 51.0 & $.59 *$ \\
\hline Físico & $56(13.8)$ & $28.6-78.6$ & .74 & 54.9 & $.52 *$ \\
\hline Meio Ambiente & $52.1(9.7)$ & $31.25-71.9$ & .69 & 48.3 & $.69 *$ \\
\hline Relações Sociais & $44.7(18.9)$ & $8.3-75$ & .66 & 43.4 & $.43^{*}$ \\
\hline Qualidade de Vida Geral & $52.5(9.6)$ & $29.3-77.3$ & - & - & - \\
\hline
\end{tabular}


A fim de estudar o efeito das características sociodemográficas, ocupacionais e do estresse em cada um dos domínios de qualidade de vida do wHoQoL-Bref (psicológico, físico, meio ambiente, relações sociais e qualidade de vida geral), foram feitos modelos de regressão linear múltipla das características, nos quais os domínios eram colocados como variáveis dependentes. $\mathrm{Na}$ tabela 3, pode-se observar que utilizar carro como meio de transporte influencia negativamente a qualidade de vida geral, o domínio físico, o domínio social e o domínio ambiental. Ainda, nota-se que ter disposição para realizar atividades de lazer influencia positivamente os domínios geral, físico e psicológico. Ter estresse tem influência negativa no domínio psicológico. O maior o tempo de deslocamento entre a moradia do residente e os locais de atuação na RM é risco para o domínio social. Para o domínio ambiental, ter tempo para lazer é um fator de proteção.

Tabela 3. Regressões lineares múltiplas da qualidade de vida e seus domínios com as variáveis sociodemográficas, ocupacionais, estresse e satisfação com o trabalho. São Paulo, 2015

\begin{tabular}{|c|c|c|c|}
\hline & Qualidade de Vida & B & IC $_{95 \%}$ \\
\hline \multirow[t]{2}{*}{ Geral } & Utilizar carro como meio de transporte & 10.4 & 3.94 a $16.8^{*}$ \\
\hline & Ter disposição para lazer & -10.85 & $-15.73 a-5.98^{* *}$ \\
\hline \multirow{2}{*}{$\begin{array}{l}\text { Domínio } \\
\text { Físico }\end{array}$} & Utilizar carro como meio de transporte & 9.33 & -0.86 a 19.52 \\
\hline & Ter disposição para lazer & -15.43 & -23.15 a $-7.72^{*}$ \\
\hline \multirow{2}{*}{$\begin{array}{l}\text { Domínio } \\
\text { Psicológico }\end{array}$} & Ter disposição para lazer & -7.51 & -14.38 a $-0.65^{*}$ \\
\hline & Ter estresse & 11.06 & 3.90 a $18.21^{*}$ \\
\hline \multirow[t]{2}{*}{$\begin{array}{l}\text { Domínio } \\
\text { Social }\end{array}$} & $\begin{array}{l}\text { Maior tempo de deslocamento para locais } \\
\text { de atuação da residência }\end{array}$ & 7.10 & 2.39 a $11.81^{*}$ \\
\hline & Utilizar carro como meio de transporte & 18.94 & 4.23 a $33.64^{*}$ \\
\hline \multirow{2}{*}{$\begin{array}{l}\text { Domínio } \\
\text { Ambiental }\end{array}$} & Ter tempo para lazer & -7.52 & -12.80 a $-2.23^{*}$ \\
\hline & Utilizar carro como meio de transporte & 8.65 & 1.11 a $16.19 *$ \\
\hline
\end{tabular}

${ }^{*} \mathrm{p}<.05 ;{ }^{* *} \mathrm{p}<.001 ; \mathrm{B}=$ Coeficiente.

\section{Discussão}

$A_{\text {quais há predominância de residentes multiprofissionais do sexo feminino, solteiros e }}^{\text {o analisar os dados, notou-se um perfil semelhante a outros estudos com residentes nos }}$ sem filhos, na faixa etária entre 22 e 35 anos $(24,25)$. Nas últimas décadas, houve intensificação da participação das mulheres na atividade econômica (26). Haddad et al. destacaram a predominância feminina em 13 cursos, de um total de 14, de graduação na área da saúde. 
Nas profissões mais encontradas no nosso estudo, os autores observaram prevalência feminina de $90 \%$ entre os estudantes de serviço social, terapia ocupacional, fonoaudiologia e nutrição (27). Desta forma, pode-se destacar a feminização da força de trabalho no setor da saúde.

Diante da observação de que 78.9 \% dos residentes estavam estressados, se faz necessário refletir a influência deste dado na saúde dos profissionais. Dias et al. afirmaram que o estresse, além de poder desencadear doenças físicas, leva a esgotamento emocional e atitudes desfavoráveis em relação ao trabalho e mudanças comportamentais para com os colegas (28). Ainda, o estresse tem relação direta com a produtividade do indivíduo, de forma que fisiologicamente a presença dele interfere na diminuição de produção de adrenalina, o que o torna apático e menos produtivo (29). A maioria dos residentes encontravam-se na fase de resistência, na qual o indivíduo automaticamente tenta lidar com os seus estressores, de modo a manter sua homeostase interna. Nesta fase, há manifestação de sintomas da esfera psicossocial, entre elas medo, isolamento social, ansiedade, impotência sexual e oscilação do apetite (30). Se os fatores estressantes forem persistentes ou em grande dimensão, o indivíduo passa para a fase de exaustão, na qual não consegue mais conviver com o estresse e pode desenvolver doenças como hipertensão arterial, depressão, ansiedade entre outros problemas (31, 32).

Para satisfação com o trabalho, a maior parte dos residentes encontrava-se no nível de satisfação intermediária. Entre os motivos de insatisfação, encontrava-se o relacionamento com os pares. Estudos destacam que o relacionamento ruim entre colegas influencia na satisfação no trabalho, o que corrobora com nossos achados. Ramires et al. identificaram que a insatisfação no aspecto relacionamento estava associada ao esgotamento profissional (33, 34). Diante dos dados apresentados, torna-se fundamental a discussão quanto estratégias de auxílio ao enfrentamento destas questões dentro do contexto da RM. Em nosso estudo, 40.5\% dos sujeitos se declararam insatisfeitos com o grau de flexibilidade e de liberdade que julgam ter em seu trabalho. O residente é um profissional que, perante seu conselho de classe e à sociedade, responde pelas ações as quais executa em seu trabalho. Por vezes, este é tratado somente como aluno, e desta forma tem sua autonomia diminuída. Sua participação no processo de trabalho deve ser ressaltada, pois ele está em treinamento e qualificação para melhor cuidar e atender as necessidades da população. De modo que é importante olhar além do conhecimento técnico-científico para valorizar também seu desenvolvimento como pessoa, para que o conhecimento profissional também seja balizado. A implantação de planos de fortalecimento das relações interpessoais no trabalho, programas específicos de promoção e prevenção da saúde física e mental dos profissionais são fundamentais para o processo de humanização das instituições de saúde e, por conseguinte, para melhoria do sistema de saúde (35). Assim, ao olhar e cuidar do residente, estamos indiretamente cuidando da população. 
Com relação à qualidade de vida, não existem pontos de corte que determinem acima ou abaixo do qual se pode identificar esta como "ruim" ou "boa" (14). Contudo, pode-se traçar um paralelo para com a população em geral. Em nosso estudo, observou-se que o domínio psicológico, que avalia se o entrevistado está satisfeito com a frequência de sentimentos negativos ou consigo mesmo e com sua aparência, entre outras, mostrou média significativamente mais elevada em relação aos demais domínios. As relações sociais apresentaram a menor média dentre os domínios. Neste item, são abordados o apoio que recebe, a satisfação com a vida social e com as pessoas do seu círculo social. Os dados do questionário de satisfação com o trabalho, mostram baixa satisfação com o relacionamento entre os pares. Todos os valores foram inferiores ao de enfermeiros de unidades de terapia intensiva e nutricionistas, fisioterapeutas e enfermeiros de um hospital universitário, exceto o meio ambiente. Ainda pode-se destacar que em todos os domínios as médias apresentadas foram inferiores quando comparados à população brasileira em geral (físico $=58.9$, psicológico $=65.9$, social $=76.2 \mathrm{e}$ meio ambiente $=59.9)(36,37)$.

Estes dados apontam para a necessidade de se ter atenção quanto à qualidade de vida dos residentes multiprofissionais. Nossos achados mostram que ter tempo e disposição para realizar atividades de lazer são fatores de proteção para qualidade de vida. Entretanto, utilizar carro para chegar aos locais de atuação da residência, presença de estresse e maior tempo de deslocamento entre a moradia do residente e os locais atuação na RM, tiveram influência negativa para esta variável. A utilização de carro como meio de transporte nas grandes cidades frequentemente enfrenta congestionamentos, que podem provocar efeitos indiretos à saúde, como estresse e ansiedade, diminuição do tempo em que o indivíduo pode realizar outras atividades como lazer, estar entre familiares ou descanso.

É importante considerar que os resultados esperados dos programas de treinamento em serviço de saúde, como é o caso das RM, são influenciados pela estrutura e logística dos mesmos. Segundo a Resolução cNRms n³, de 4 de maio de 2010, os Programas de Residência Multiprofissional em Saúde e em Área Profissional da Saúde devem ter a duração mínima de dois anos, com uma carga horária mínima total de 5760 (cinco mil, setecentos e sessenta) horas, o que equivale a uma carga de 60 horas semanais (38). Em um momento cultural no mundo do trabalho em que os sindicatos trabalhistas defendem a diminuição da jornada de trabalho principalmente devido à sobrecarga à saúde do trabalhador, a carga horária estabelecida pelos programas RM ultrapassa aquela definida pelas organizações competentes das profissões abrangidas. Afim de atender as necessidades de saúde do sistema brasileiro, o treinamento e a qualificação do profissional de saúde têm passado por reestruturações, de modo que as estratégias implementadas visam a integralidade do cuidado. Tais mudanças, quando bem planejadas e desenvolvidas, podem ser positivas na atuação do profissional e, por conseguinte, na assistência à população. 
A prevalência do estresse nos residentes multiprofissionais foi alta, a maioria encontravase na fase de resistência seguida das fases de alerta e exaustão. Houve predominância de satisfação parcial em relação à satisfação com o trabalho de modo geral. A maior proporção de residentes satisfeitos foi com o quanto os programas absorvem suas potencialidades, suas estruturas organizacionais e implementação de mudanças e inovações. Em oposição, estavam insatisfeitos com o relacionamento com outras pessoas, com a flexibilidade e com liberdade no trabalho. A qualidade de vida dos residentes multiprofissionais aqui estudados avaliada pelo instrumento wHoQoL-bref apresentou as seguintes médias: domínio físico = 56 ; domínio psicológico $=58.2$; meio ambiente $=52.1$; relações sociais $=44.7$ e qualidade de vida geral $=52.5$. 0 estresse e a mobilidade urbana contribuíram negativamente para a Qv, por outro o lazer teve influência positiva.

Vale considerar que este estudo foi transversal, não possibilitando fazer afirmações de causalidade. Apesar de estudarmos os diversos programas de RM, os resultados referem-se a uma faculdade de uma universidade pública do estado de São Paulo.

Sabe-se que a qualidade de vida envolve outros fatores além do trabalho, contudo, estes profissionais dedicam boa parte de suas horas semanais à residência, de modo que pode-se sugerir que as diretrizes dos programas Rм estudados sejam discutidas a fim de fortalecer o relacionamento entre os profissionais, diminuir o estresse apresentado entre os outros fatores apontados como prejudiciais à saúde destes, o que pode favorecer também os serviços de saúde, nos quais eles estão inseridos, na medida em que indivíduos satisfeitos podem influenciar positivamente a qualidade da assistência prestada. Em geral, poucos estudos contemplam a saúde do residente multiprofissional, dessa forma sugere-se que sejam realizadas mais pesquisas a fim de explorar a saúde, o trabalho, o bem-estar físico e mental desses trabalhadores.

\section{Agradecimentos}

Eesquisa foi apresentada como Trabalho de Conclusão de Residência da Faculdade de $_{\text {mespos }}$ Medicina da usp - Departamento de Fisioterapia, Fonoaudiologia e Terapia Ocupacional como requisito básico para a conclusão da Residência Multiprofissional em Promoção da Saúde e Cuidado na Atenção Hospitalar - Área de Concentração de Saúde Coletiva com Ênfase em Saúde e Trabalho. São Paulo - Brasil, 2015. 


\section{Conflitos de interesse}

eclaramos não haver conflito de interesses.

\section{Referências}

1. Rosa SD, Roseli El. Residência multiprofissional em saúde e pós-graduação lato sensu no Brasil: apontamentos históricos. Trab Edu Saúde. 2010;7(3):479-98. Dor: 10.1590/S198177462009000300006

2. Portal Ministério da Educação e Cultura. Residência Multiprofissional. Resolução cNs $n^{\circ}$ 287/1998 [internet]. (2014) [citado 2014 nov 15]. Disponível em: http://portal.mec. gov.br/index.php?option=com_content\&id=12501\&Itemid=813

3. Feuerwerker L. Mudanças na educação médica e residência médica no Brasil. Interface (Botucatu). 1998;2(3):51-71. Dor: 10.1590/S1414-32831998000200005

4. Nogueira-Martins LA. Qualidade de vida dos médicos residentes: revisão de estudos brasileiros. Cad ABEM. 2010;6:12-8.

5. Nogueira-Martins LA, Jorge MR. Natureza e magnitude do estresse na residência médica. Rev Assoc Med Bras. 1998;44(1):28-34. Dor: 10.1590/S0104-42301998000100006

6. Lourenção LG, Moscardini AC, Soler ZASG. Saúde e qualidade de vida de médicos residentes. Rev Assoc Med Bras. 2010;56(1):81-91. Dor: 10.1590/S0104-42302010000100021

7. Instituto Brasileiro de Geografia e Estatística. Pesquisa nacional de amostras de domicílios contínua, 2013 - Notas Metodológicas [internet]. [citado 2015 nov 10]. Disponível em: http:www.ibge.gov.br

8. Ministério do Trabalho e Emprego, Portal do Trabalho e Emprego. Códigos, títulos e descrições Classificação Brasileira de Ocupações [internet] 2010. [citado 2015 nov 10]. Disponível em: http://www.mtecbo.gov.br/cbosite/pages/pesquisas/BuscaPorTitulo.jsf

9. Junior AF, Andrade DR, Andrade E, Braggion GF, Oliveira LC, Pardini R, et al. Validação do questionário internacional de nível de atividade física (IPAQ-versão 6): estudo piloto em adultos jovens brasileiros. Rev. Bras. Ciên. e Mov. Brasília. 2001;9(3):39-44.

10. Kluthcovsky ACGC, Kluthcovsky FA. O whoQoL-bref, um instrumento para avaliar qualidade de vida: uma revisão sistemática. Rev Psiquiatr Rio Gd Sul. 2009;31(3):supl. dor: 10.1590/S0101-81082009000400007

11. Fleck MPA, Louzada S, Xavier M, Chachamovich E, Vieira G, Santos L, et al. Application of the Portuguese version of the abbreviated instrument of quality life wHOQOL-bref. Rev Saúde Pública. 2000;34(2):178-83. Dor: 10.1590/S0034-89102000000200012

12. Santos ECM, França JI, Lopes F. Qualidade de vida de pessoas vivendo com HIV/AIDs em São Paulo. Rev Saúde Pública. 2007;41(2):64-71. Dor: 10.1590/S0034-89102007000900011

13. Braga MCP, Casella MA, Campos MLN, Paiva SP. Qualidade de vida medida pelo wHoQoL-bref: estudo com idosos residentes em Juiz de Fora/MG. Rev APs 2011;14(1):93-100. 
14. Pereira RJ, Cotta RMM, Franceschini SCC, Ribeiro RCL, Sampaio RF, Priore SE, et al. Contribuição dos domínios físico, social, psicológico e ambiental para a qualidade de vida global de idosos. Rev Psiquiatr Rio Gd Sul. 2006;28(1):27-38. Dor: 10.1590/S010181082006000100005

15. Abdel-Khalek AM. Quality of life, subjective well-being, and religiosity in Muslim college students. Qual Life Res. 2010;19(8):1133-43. Dor: 10.1007/s11136-010-9676-7

16. Lipp MEN. Manual do inventário de sintomas de stress para adultos de Lipp (ISsL). $3^{\mathrm{a}}$ ed. São Paulo: Casa do Psicólogo; 2000.

17. Martinez MC, Paraguay AIBB, Latorre MRDO. Relação entre satisfação com aspectos psicossociais e saúde dos trabalhadores. Rev Saúde Pública 2004;38(1):55-61. DoI: 10.1590/ S0034-89102004000100008

18. Sadir MA, Bignotto MM, Lipp MEN. Stress e qualidade de vida: influência de algumas variáveis pessoais. Paidéia (Ribeirão Preto). 2010;20(45):73-81. Dor: 10.1590/S0103863X2010000100010

19. Robertson IT, Cooper CL, Williams J, Williams J. The validity of the occupational stress indicator. Work \& Stress 1990;4(1):29-39. Dor: 10.1080/02678379008256962

20. Swan JA, Renault De Moraes LF, Cooper CL Developing the occupational stress indicator (osI) for use in Brazil: a report on the reliability and validity of the translated osi. Stress Medicine 1993;9(4):247-53. Dor: 10.1002/smi.2460090407

21. Baptista MN, Souza MS, Degenhardt I, Nicoletti IG. Escala de Suporte Laboral (Esul) e Escala de Satisfação no Trabalho: evidências de validade. Aval Psicol. 2010;9(3): 479-88.

22. Barros Junior JC, Cesário M, Morraye MA, de Paula Júnior JD. Avaliação de satisfação no trabalho em uma mine, adora brasileira. Investigação 2011;11(2):29-36.

23. Conselho Nacional de Saúde. Comissão de Ética em Pesquisa (Brasil). Resolução No 466 , (2012 dez 12). Diário Oficial da União (Brasília) 2012. p. 59.

24. Goulart CT, Silva RM, Bolzan MED, Guido LD. Perfil sociodemográfico e acadêmico dos residentes multiprofissionais de uma universidade pública. Rev Rene. 2012;13(1):178-86.

25. de Azevedo-Guido L, da Silva RM, Goulart CT, de Oliveira-Bolzan ME, Lopes LFD. Síndrome de Burnout em residentes multiprofissionais de uma universidade pública. Rev Esc Enferm UsP. 2012;46(6):1477-83. Dor: 10.1590/S0080-62342012000600027

26. Hoffmann R, Leone ET. Participação da mulher no mercado de trabalho e desigualdade da renda domiciliar per capita no Brasil: 1981-2002. Nova Economia 2004;14(2):35-58.

27. Haddad AE. A trajetória dos cursos de graduação na saúde: 1991-2004. Brasília: Instituto Nacional de Estudos e Pesquisas Educacionais Anísio Teixeira [internet]. 2006 [citado 2015 dez 01]. Disponível em: http://bvsms.saude.gov.br/bvs/publicacoes/Texto_de_ Referencia.pdf

28. Dias LG, Nogueira MM, Dutra GO, Mazon B, de Ávila LC. Characterization and ways to fight stress in professional nursing care pre-hospital. Rev pesqui cuid fundam 2011;31(1): 1582-93.

29. Pafaro RC. De Martino MMF. Estudo do estresse do enfermeiro com dupla jornada de trabalho em um hospital de oncologia pediátrica de Campinas. Rev Esc Enferm usp . 2004; 38(2):152-60. DoI: 10.1590/S0080-62342004000200005 
30. Katsurayama M, Gomes NM, Becker MAD, Santos MC, Makimoto FH, Santana LLO. Avaliação dos níveis de estresse psicológico em médicos residentes e não residentes de hospitais universitários. Psicol hosp (São Paulo) . 2011;9(1):75-96.

31. Short KH, Johnston C. Stress, maternal distress, and children's adjustment following immigration: The buffering role of social support. J Consult Clin Psychol . 1997; 65(3):494-503. Dor: 10.1037/0022-006X.65.3.494

32. Lipp MEN. Stress: conceitos básicos. In: Lipp MEN, organizadora. Pesquisas sobre stress no Brasil: sáude, ocupações e grupos de risco. Campinas (SP): Papirus; 1996. p. 17-31.

33. Elovainio M, Kivimãki M, Steen N, Kalliomãki-Levanto T. Organizational and individual factors affecting mental health and job satisfaction: A multilevel analysis of job control and personality. J Occup Health Psychol. 2000;5(2):269-77. Dor: 10.1037/1076-8998.5.2.269

34. Ramirez AJ, Graham J, Richards MA, Gregory WM, Cull A. Mental health of hospital consultants: the effects of stress and satisfaction at work. Lancet .1996;347(9003):724-8. Dor: 10.1016/S0140-6736(96)90077-X

35. Damas KCA, Munari DB, Siqueira KM. Cuidando do cuidador: reflexões sobre o aprendizado dessa habilidade. Rev Eletrônica Enferm. 2006;6(2):272-78.

36. Spiller APM, Dyniewicz AM, Slomp MGFS. Qualidade de vida de profissionais da saúde em hospital universitário. Cogitare enferm. 2008;13(1). Dor: 10.5380/ce.v13i1.11965

37. Cruz LN, Polanczyk CA, Camey SA, Hoffmann JF, Fleck MP. Quality of life in Brazil: normative values for the wHoQoL-bref in a southern general population sample. Qual life res. 2011;20(7):1123-9. Dor: 10.1007/s11136-011-9845-3

38. Ministério da Educação (Brasil). Resolução cnRMs n³. (2010 mai 04). Diário Oficial da União 5 maio 2010; Seção 1, p. 14-15. 